\title{
Pneumothorax Found during Health Check-up as a Manifestation of Thoracic Endometriosis
}

\author{
Atsushi Imai ${ }^{1 *}$, Satoshi Ichigo ${ }^{1}$, Hiroshi Takagi ${ }^{1}$, Kazutoshi Matsunami ${ }^{1}$, Naoki Nishina ${ }^{2}$ and \\ Toshio Kasugai ${ }^{2}$
}

${ }^{1}$ Department of Obstetrics and Gynecology, Matsunami General Hospital, Japan

${ }^{2}$ Department of Thoracic Surgery, Matsunami General Hospital, Japan

*Corresponding author: Atsushi Imai, MD, PhD, Department of Obstetrics and Gynecology, Matsunami General Hospital, 185-1

Dendai, Kasamatsu, Gifu 501-6062, Japan

\section{ARTICLE INFO}

Received: 幽 October 01, 2020

Published: October 13, 2020

Citation: Imai A, Ichigo S, Takagi, $\mathrm{H}$, Matsunami K, Naoki N, Kasugai T. Pneumothorax Found during Health Check-up as a Manifestation of Thoracic Endometriosis. Biomed J Sci \& Tech Res 31(1)-2020. BJSTR. MS.ID.005056.

\section{ABSTRACT}

Women of reproductive age group who experience the catamenial pneumothorax and temporal respiratory symptoms may lead the physician to the possibility of thoracic endometriosis. We herein describe a 38-year-old woman who initially visited the medical checkup-institute to discover problems that may be harmful to the future health of asymptomatic examinees. Chest radiography showed a right-sided pneumothorax. On further questioning the patient felt uncomfortable in the right back of chest that coincided with her current menstrual period since past 1 year. A presumptive diagnosis of thoracic endometriosis prompted us to submit her to video-assisted thoracoscopic surgery to obtain tissue for pathologic examination. Thoracoscopy showed extensive endometriosislike lesions involving the right posterior hemidiaphragm. A pathologic examination of the resected tissue confirmed the very rare diagnosis of thoracic endometriosis.

\section{Introduction}

Endometriosis is defined as growth of endometrial glands and stroma outside the endometrial cavity [1,2]. The incidence of endometriosis is $15 \%$ or more among reproductive-age women, and about $10-15 \%$ of women with endometriosis have extrapelvic disease: however, the exact prevalence of thoracic endometriosis is unknow [3-5]. Weighed clinical suspicion carries to diagnosis of thoracic endometriosis. The clinical manifestations of thoracic endometriosis can be variable, with many patients being asymptomatic. The common symptoms include chest pain, dyspnea, or cough around the time of her menstrual cycle. This report mentions a very rare case of thoracic endometriosis presenting with pneumothorax accidentally found during health check-up.

\section{Case Report}

A 38-year-old woman, gravida 1, para 1, was initially visited the unique institute (namely Ningen Dock in Japan) of complete medical check-up to discover problems that may be harmful to the future health of asymptomatic examinees. Chest radiography revealed a right-sided pneumothorax (Figure 1a). On further questioning, she felt uncomfortable in the right back of chest that coincided with her current menstrual period since past 1 year. She reported regular menstrual cycles, and never had dysmenorrhea or dyspareunia. She denied fevers, chills, shortness of breath, chest pain, fever, or chills. Although it is rare, these circumstantial situations led us to a presumptive diagnosis of thoracic endometriosis. We decided to proceed with videoassisted thoracoscopic surgery to obtain a definitive diagnosis. Thoracoscopy revealed extensive endometriosis-like foci involving the right posterior hemidiaphragm (Figure 1b). The involved area was fulgurated and resected, and the diaphragm was repaired using a direct suture. A pathologic examination of the resected tissue confirmed endometriosis. Subsequent MRI of her pelvis detected endometriosis cyst measuring $38 \times 40 \mathrm{~mm}$ in the left ovary. To date, she is using gonadotropin-releasing hormone analogues for 6 months and remains asymptomatic. 

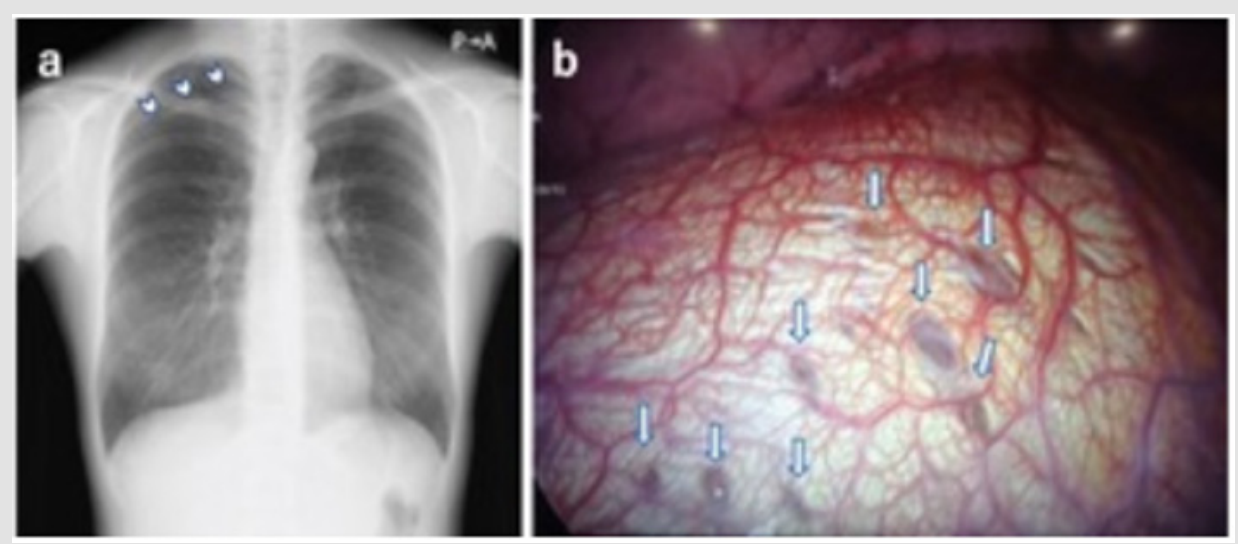

Figure 1:

(a) Chest X-ray demonstrating a right-sided pneumothorax (arrowheads) at the health check-up examination.

(b) Thoracoscopic aspect of diffuse endometriosis involving pleural surface of the diaphragm (arrows).

\section{Comments}

Diagnosis of thoracic endometriosis results heavily from suspicion [3-5]. Most patients will present with symptoms consistent with catamenial pneumothorax: shortness of breath, cough, and pleurisy. In our case, however, pneumothorax found by chance during medical check-up led us the possibility of thoracic endometriosis, taken together with retrospective questioning on cyclic thoracic uncomfortable complaint. Chest radiograph, CT, MRI, thoracentesis, and bronchoscopy have been deemed useful in evaluating thoracic endometriosis [3-5]. However, video-assisted thoracoscopic surgery remains the most accepted options for both definitive diagnosis and surgical treatment. The majority of cases of thoracic endometriosis occur in the third and fourth decade of life, compared with younger peak incidence for pelvic endometriosis [6-8]. Right-sided pneumothorax is seen in most instances. In thoracic endometriosis it assumes even more importance due to the fact that extra pelvic endometriosis usually has concomitant pelvic endometriosis [3-5]. Although the patient is still hormonal suppression, the incidence of endometriosis (15\% or higher) and the likelihood of its progression prompted us to report the timely diagnosis followed with adequate medical managements. This study can be suggestive as it not only revealed a very rare diagnosis, but also highlight that a highly suspected thoracic endometriosis should be considered in women of a menstruating age.

\section{ISSN: 2574-1241}

DOI: 10.26717/BJSTR.2020.31.005056

Atsushi Imai. Biomed J Sci \& Tech Res

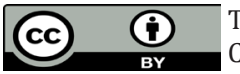

This work is licensed under Creative Commons Attribution 4.0 License

Submission Link: https://biomedres.us/submit-manuscript.php

\section{Disclosure Statement}

The authors declare no conflict of interests regarding the publication of this report.

\section{References}

1. Giudice LC (2010) Endometriosis. N Engl J Med 362: 2389-2398..

2. Greene AD, Lang SA, Kendziorski JA, Sroga Rios JM, Herzog TJ, et al. (2016) Endometriosis: where are we and where are we going? Reproduction 152: R63-R78.

3. Azizad Pinto P, Clarke D (2014) Thoracic endometriosis syndrome: Case report and review of the literature. Perm J 18: 61-65.

4. Nezhat C, Lindheim SR, Backhus L, Vu M, Vang N, et al. (2019) Thoracic Endometriosis Syndrome: A Review of Diagnosis and Management. JSLS 23(3): e2019.00029.

5. Larraín D, Suárez F, Braun H, Chapochnick J, Diaz L, et al. (2018) Thoracic and diaphragmatic endometriosis: Single-institution experience using novel, broadened diagnostic criteria. J Turk Ger Gynecol Assoc 19(3): 116-121.

6. Jessica Gates, Akhilesh Sharma, Arunesh Kumar (2018) Rare case of thoracic endometriosis presenting with lung nodules and pneumothorax. BMJ Case Rep.

7. Joseph J, Sahn SA (1996) Thoracic endometriosis syndrome: New observations from an analysis of 110 cases. Am J Med 100: 164-170.

8. Channabasavaiah AD, Joseph JV (2010) Thoracic endometriosis: Revisiting the association between clinical presentation and thoracic pathology based on thoracoscopic findings in 110 patients. Medicine 89:183-188

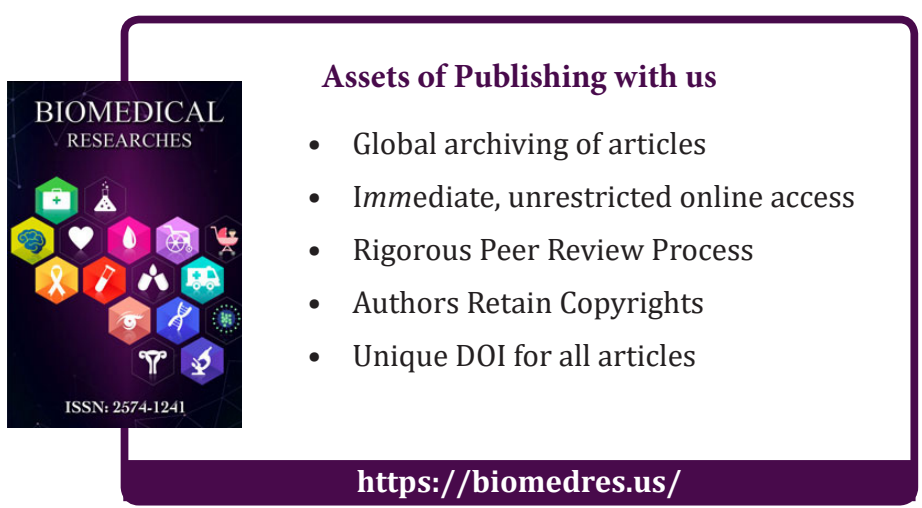

